\title{
Risks and challenges in multiple pregnancy
}

\author{
Aditi Agarwal*, Arti Sharma, Neeta Bansal, Neha Panwar, Shweta Singh, Swati Kohli
}

\begin{abstract}
Department of Obstetrics and Gynaecology, Shri Guru Ram Rai Institute of Medical and Health Sciences, Dehradun, Uttarakhand, India
\end{abstract}

Received: 28 September 2021

Accepted: 28 October 2021

\section{*Correspondence:}

Dr. Aditi Agarwal,

E-mail: aditiagarwal47@gmail.com

Copyright: (C) the author(s), publisher and licensee Medip Academy. This is an open-access article distributed under the terms of the Creative Commons Attribution Non-Commercial License, which permits unrestricted non-commercial use, distribution, and reproduction in any medium, provided the original work is properly cited.

\section{ABSTRACT}

Because of breakthroughs in reproductive medicine, multiple pregnancies have become more common in the industrialized world. As a result, obstetric care for women with multiple pregnancies and neonatal care for the same has become more demanding. Multifetal pregnancies are linked to an elevated risk for both the mother and child. There is increased incidence of obstetric complications such as spontaneous abortion, hypertensive disorders, placenta previa, and fetal malformations. Perinatal outcome is also affected which is attributable to increased incidence of fetal complications like prematurity, congenital malformations, growth discordance and cord accidents. We are presenting 2 cases, one of MCMA twin pregnancy with fetal congenital anomaly and another of a triplet pregnancy and their perinatal outcomes.

Keywords: Multifetal pregnancies, Congenital malformations, MCMA twin pregnancy

\section{INTRODUCTION}

Multifetal pregnancies may result from two or more fertilization events, from a single fertilization followed by an erroneous splitting of the zygote, or from a combination of both. Such pregnancies are associated with increased risk for both the mother and the child. Fueled largely by infertility therapy, both the rate and number of twin and higher order multifetal birth have increased dramatically since 1980. The overall increase in the prevalence of multifetal births is concern because the corresponding increase in the rate of preterm birth and congenital anomalies compromises neonatal survival and increases the risk of lifelong disability.

Monozygotic twin gestations account for less than 5\% of monozygotic twin pregnancies and $1 \%$ of all twin pregnancies. ${ }^{1}$ When compared to singletons, the incidence of congenital abnormalities is significantly higher in multifetal pregnancy. According to multiple studies, the malformation rate in monochorionic twins was almost twice that of dichorionic twin gestations. This increase has been attributed to the high incidence of structural defects in monozygotic twins. Twins and triplets have a perinatal mortality rate that is 4 to 11 times that of singletons. ${ }^{2}$

Ovulation induction, in vitro fertilization, and related methods of assisted reproductive technology have been linked to increased risk of triplet and higher multiple pregnancies. ${ }^{3}$ Twin and triplet gestation are associated with increased incidence of maternal complications like gestational hypertension, preclampsia and preterm labor along with an adverse perinatal outcome as compared to that of a singleton pregnancy.

In this study, we report 2 cases of multifetal gestation, one of MCMA pregnancy and another of triplet pregnancy and their perinatal outcomes.

\section{CASE REPORT}

\section{Case 1}

A 20-year-old primigravida presented to our hospital for routine antenatal checkup in early pregnancy. She conceived spontaneously after 1 year of married life. Her 
level I (NT NB scan) was suggestive of twin live gestation 12 weeks 3 days and a single amniotic cavity suggesting monochorionic and monoamniotic pregnancy.

A subsequent level II scan was done which showed features of congenital diaphragmatic hernia in fetus A. Fetus B had no gross congenital anomalies.

She was followed regularly throughout the pregnancy as she was a booked case. At 35 weeks 3 days of gestation, a caesarean section was done in view of monochorionic monoamniotic (MCMA) pregnancy after a course of steroids.

Intra operatively, a single amniotic bag was ruptured and clear liquor was drained. $1^{\text {st }}$ twin, male, was delivered by cephalic presentation, did not cry immediately after birth. Weight was $2.3 \mathrm{~kg}$ and Apgar of 4 at 1 minute and 2 at 5 minutes was given. The baby had congenital diaphragmatic hernia which was diagnosed antenatally and had respiratory distress at birth and hence, was immediately intubated. $2^{\text {nd }}$ twin, male, delivered by assisted breech extraction had immediate cry after birth. Weight was $2 \mathrm{~kg}$ and Apgar of 7 at 1 minutes and 8 at 5 minutes was given. Placenta was single with 2 cord insertions and a true knot was present (Figure 1). Perinatal Outcome: $1^{\text {st }}$ baby with congenital diaphragmatic hernia had respiratory distress and was kept on ventilatory support for one day and the baby died after one day of life. $2^{\text {nd }}$ baby had an uneventful perinatal period and was mother side just after birth and was exclusively breastfed. Post operative period of the patient was uneventful.

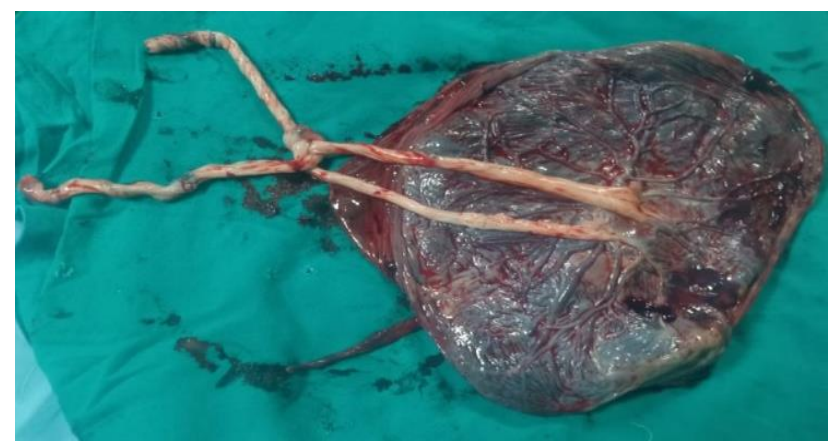

Figure 1: Placenta of MCMA pregnancy with a true knot.

\section{Case 2}

A 40-year-old patient, G3A2, presented to our antenatal OPD at 34 weeks gestation with triplet pregnancy with preclampsia. She had been married for past 15 years and treated for secondary infertility by IVF. She was referred in view of high-risk triplet pregnancy with raised blood pressure. On examination she had marked Bilateral pitting type of pedal oedema, with no signs of pallor, icterus, cyanosis, clubbing or lymphadenopathy. Her Blood Pressure was $160 / 100 \mathrm{~mm}$ of $\mathrm{Hg}$ at the time of presentation.
Her sonographic report at 12 weeks scan shows gravid uterus with triple viable foetuses with CGA of first fetus 12 weeks, CGA of second fetus was 11 weeks +6 days and CGA of third fetus was 11 weeks +6 days.

Her level II scan was done showing triplets with CGA of first twin 18 weeks +6 days, CGA of second fetus 18 weeks +2 days and CGA of third fetus 19 weeks, with placenta anterior, amniotic fluid adequate without any anomalies.

A repeat scan was done at our hospital which shows triplet alive intrauterine foetuses seen with $\mathrm{F}$-A in breech with CGA 33 weeks +6 days, F-B in cephalic presentation with CGA 34 weeks +1 day and F-C in transverse lie with CGA 32 weeks +4 days, with good cardiac activity. Placenta is anterior, away from internal OS, separate delineation of placenta could not be done, intertwin membrane seen with adequate liquor for the gestational age. Mean EFW of triplet A-was $2125 \mathrm{gm}$, EFW of triplet B $2189 \mathrm{gm}$ and EFW of triplet C was $1806 \mathrm{gm}$.

Keeping in mind the risk of adverse events, a decision for caesarean section was taken at gestational age of 34 weeks +5 days in view of triplet pregnancy with $1^{\text {st }}$ triplet in breech.

\section{Intraoperative findings}

An alive triplet (I)-baby girl delivered as breech with clear liquor, triplet (II) baby girl delivered as vertex with meconium-stained liquor and triplet (III) baby girl as breech with meconium-stained liquor, weighing $1980 \mathrm{gm}$, $1830 \mathrm{gm}$ and $1625 \mathrm{gm}$ respectively. All triplets cried Immediately after birth. A large placenta with 3 cord insertions was seen (Figure 2).

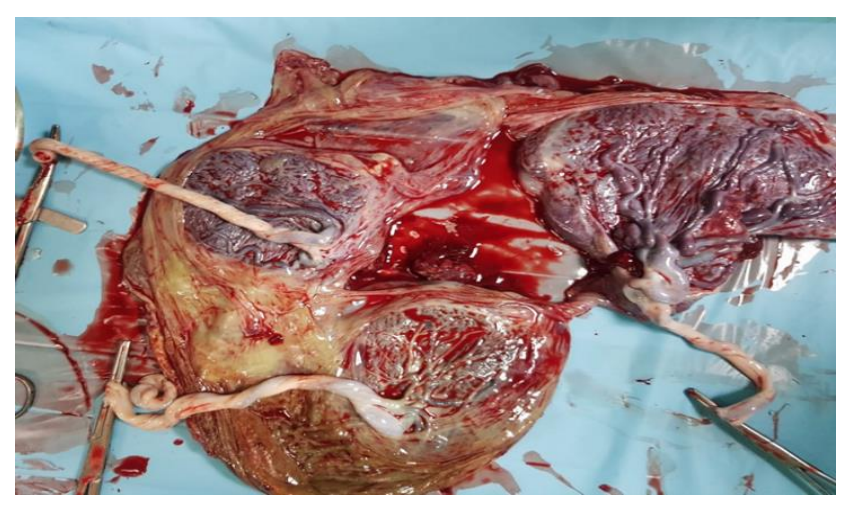

Figure 2: Large placenta of triplet pregnancy with 3 cord insertions.

\section{Perinatal outcome}

All three triplets had an uneventful perinatal period with no history of admission to NICU. Post operative period of the patient was uneventful. 


\section{DISCUSSION}

MCMA twin gestations are derived from a single blastocyst in which the zygotic division takes place for more than 8 days after fertilization. ${ }^{4}$ In these pregnancies, the fetuses are at high risk due to the shared vascular areas as well as cord accidents and birth trauma. MCMA twin gestations have been therefore associated with high perinatal mortality rates. Umbilical cord entanglements and knots, twin-to-twin transfusion syndrome, congenital anomalies, prematurity and intertwin locking during labour are responsible for their high perinatal morbidity and mortality. ${ }^{5}$ MCMA twins carry an additional risk for perinatal death due to twin-related congenital anomalies and cord-related complications, resulting in about $15 \%$ of deaths after 20 weeks of pregnancy. ${ }^{5}$

In a study conducted by Santema et al they concluded that the anticipated perinatal outcome in triplet and twin pregnancies may be used to counsel women with a triplet pregnancy considering selective reduction to twins and all methods of assisted reproduction should aim at prevention of multifetal gestation. ${ }^{6}$

In another study conducted by Glinianaia et al they concluded that in MCMA twins surviving beyond 24 weeks of gestation, there was a higher survival rate compared to previous decades presumably due to early diagnosis, close surveillance and elective birth around 3234 weeks of gestation. High perinatal mortality at early gestations was mainly attributed to extreme prematurity due to preterm spontaneous labour. ${ }^{7}$

\section{CONCLUSION}

Multifetal gestations are at increased risk of adverse perinatal outcomes compared with singleton pregnancies. Perinatal survival of twins and triplets have improved compared with that in previous decades, presumably due to early diagnosis and careful and close fetal surveillance.

Funding: No funding sources

Conflict of interest: None declared

Ethical approval: Not required

\section{REFERENCES}

1. Cordero L, Franco A, Joy SD. Monochorionic Monoamniotic twins: neonatal outcome. J Perinatol. 2006;26:170-5.

2. Benson RC. Current Obstetric and Gynaecologic Diagnosis and Treatment. $4^{\text {th }}$ ed, Lange Medical Publications; California: Multiple pregnancy. 1982;755-63.

3. Levene MI, Wild J, Steer P. Higher multiple births and the modern management of infertility in Britain. Br J Obstet Gynaecol. 1992;99:607-13.

4. Benirschke K. The biology of twinning process: how placentation influences outcome. Semin Perinatol. 1995; 19:342-50.

5. Carr SR, Aranson MP, Coustan DR. Survival rates of monoamniotic twins do not decrease after 30 weeks gestation. Am J Obstet Gynecol. 1993;168:601-4.

6. Santema JG, Bourdrez P, Wallenburg HCS. Maternal and perinatal complications in triplet compared with twin pregnancy. Eur J Obstetr Gynecol Reproduct Biol. 1995;60(2):143-7.

7. Glinianaia SV, Rankin J, Khalil A, Binder J, Waring G, Sturgiss SN, Hannon T. Prevalence, antenatal management and perinatal outcomes of monochorionic monoamniotic twin pregnancies: a collaborative multicentre study in England, 20002013. Ultrasound in Obstetr Gynecol. 2018.

Cite this article as: Agarwal A, Sharma A, Bansal N, Panwar N, Singh S, Kohli S. Risks and challenges in multiple pregnancy. Int J Reprod Contracept Obstet Gynecol 2021;10:4578-80. 